\title{
MULTIPLIER IDEALS, V-FILTRATIONS AND TRANSVERSAL SECTIONS
}

\author{
A. DIMCA, PH. MAISONOBE, M. SAITO, AND T. TORRELLI
}

\begin{abstract}
We show that the restriction to a smooth transversal section commutes to the computation of multiplier ideals and V-filtrations. As an application we prove the constancy of the spectrum along any stratum of a Whitney regular stratification.
\end{abstract}

\section{INTRODUCTION}

Let $X$ be a complex $n$-dimensional manifold and $D \subset X$ an effective divisor defined by a holomorphic function $f$. To put the results of this paper in their proper perspective, we start by recalling the local topological triviality of Whitney regular stratifications, see for details [9], [12].

If $\mathcal{S}$ is a Whitney regular stratification of the reduced divisor $D_{\text {red }}$ and $x_{0} \in D$ belongs to a (connected) stratum $S \in \mathcal{S}$ with $d=\operatorname{dim} S>0$, then the local topology of the pair $(X, D)$ at the point $x_{0}$ is given by the product $(T, T \cap D) \times(S, 0)$, where $\left(T, x_{0}\right)$ is a smooth transversal to $S$ at $x_{0}$, i.e. $\operatorname{dim} T=n-d$ and $T \pitchfork S$. In other words, the local topology of the pair $(X, D)$ is constant along the stratum $S$.

In terms of constructible sheaves, the topology of the pair $(X, D)$ is described by the vanishing cycle sheaf complex $\phi_{f}\left(\mathbb{Q}_{X}\right) \in D_{c}^{b}\left(\mathbb{Q}_{D}\right)$, see for instance [10]. If $i_{T}: T \rightarrow X$ denotes the inclusion of the transversal $T$ above, then there is an isomorphism

$$
\phi_{f \circ i_{T}}\left(\mathbb{Q}_{T}\right)=i_{T}^{-1} \phi_{f}\left(\mathbb{Q}_{X}\right)
$$

in the derived category $D_{c}^{b}\left(\mathbb{Q}_{T \cap D}\right)$, see for instance [38, Lemma 4.3.4, p. 265. In fact this isomorphism holds for any $\mathcal{S}$-constructible complex $\mathcal{C} \in D_{c}^{b}\left(\mathbb{Q}_{X}\right)$, namely

$$
\phi_{f \circ i_{T}}\left(i_{T}^{-1} \mathcal{C}\right)=i_{T}^{-1} \phi_{f}(\mathcal{C})
$$

This more general view-point allows us, in particular, to reduce the case of an arbitrary divisor $D$ to the case of a smooth hypersurface $H$, via the following basic construction. Set $X^{\prime}=X \times \mathbb{C}$ and let $i_{f}: X \rightarrow X^{\prime}$ be the graph embedding $x \mapsto(x, f(x))$. If $t$ denotes the coordinate on $\mathbb{C}$, then one has

$$
\phi_{t}\left(i_{f *} \mathcal{C}\right)=i_{f *} \phi_{f}(\mathcal{C}) .
$$

2000 Mathematics Subject Classification. Primary 14B05, 32S35; Secondary 32S30, 32S40, $32 \mathrm{~S} 60$.

Key words and phrases. multipler ideal, V-filtration, spectrum, Whitney regular stratification. 
Indeed, $i_{f}$ is proper and $\phi_{f}$ commutes with proper direct images, see for instance [10], p. 109.

In this paper we prove two analogs of the above well-known properties.

Theorem 1.1. Let $D: f=0$ be a smooth divisor in $X$ and let $i_{T}: T \rightarrow X$ be the inclusion of a closed submanifold which is transversal to D. Assume that $M$ is a regular holonomic $\mathcal{D}_{X}$-module such that $T$ is non-characteristic for $M$ and for $M(* D)$. Let $V$ denote the Kashiwara-Malgrange filtration of $M$ along the hypersurface $D$ and also the Kashiwara-Malgrange filtration of the restriction $i_{T}^{*} M$ along the hypersurface $T \cap D$. Then, for any $\alpha \in \mathbb{C}$, the following hold:

(i) $i_{T}^{*}\left(V^{\alpha} M\right)=V^{\alpha} i_{T}^{*} M ;$ (ii) $i_{T}^{*}\left(G r_{V}^{\alpha} M\right)=G r_{V}^{\alpha} i_{T}^{*} M$.

In particular, $i_{T}^{*}\left(\psi_{f} M\right)=\psi_{f \mid T}\left(i_{T}^{*} M\right)$ and $i_{T}^{*}\left(\phi_{f} M\right)=\phi_{f \mid T}\left(i_{T}^{*} M\right)$.

For more details on the notions involved in this theorem we refer the reader to the next section. We mention here that the non-characteristic condition is a generalization of the transversality condition used in (1.1) and (1.2) which implies that the analytic pull-back $i_{T}^{*}(M)$ coincides with the left derived pull-back $\mathbb{L} i_{T}^{*}(M)$, see Proposition 2.5. Moreover, the V-filtration is the necessary ingredient to construct a theory of vanishing cycles for the regular holonomic $\mathcal{D}_{X}$-modules, see [29], [18, [32, 26] for a synthesis. For other applications of Theorem 1.1 to the monodromy and Bernstein polynomials of families of hypersurface singularities, see [28].

To state the second result, we return to the general case, i.e. $D$ is an effective divisor on $X$ defined by a holomorphic function $f$. The family of multiplier ideals $\{\mathcal{I}(\alpha D)\}_{\alpha \in \mathbb{Q}}$ associated to $D$ is a decreasing family of ideals in the structure sheaf $\mathcal{O}_{X}$ which encodes a lot of the algebraic geometry of the pair $(X, D)$, see [8], 22], [23] for more on this beautiful and very active subject. Using the deep relation between multiplier ideals and V-filtrations established in [7] and Theorem [1.1 above, we obtain the following.

Theorem 1.2. Assume $T$ is transversal to any stratum of a Whitney regular stratification of the reduced divisor $D_{\text {red }}$, or more generally, $T$ is non-characteristic for the regular holonomic $\mathcal{D}_{X}$-module $\mathcal{O}_{X}(* D)$. Then for $\alpha \in \mathbb{Q}$, we have a canonical isomorphism

$$
i_{T}^{*} \mathcal{I}(\alpha D)=\mathcal{I}(\alpha(D \cap T))
$$

compatible with the inclusions $\mathcal{I}(\alpha D) \rightarrow \mathcal{I}\left(\alpha^{\prime} D\right)$ and $\mathcal{I}(\alpha(D \cap T)) \rightarrow \mathcal{I}\left(\alpha^{\prime}(D \cap T)\right)$ for $\alpha>\alpha^{\prime}$, where the isomorphism for $\alpha \leq 0$ is the natural isomorphism $i_{T}^{*} \mathcal{O}_{X}=\mathcal{O}_{T}$.

Note that the multiplier ideals $\mathcal{I}(\alpha D)$ are essentially given by the filtration $\mathcal{J}(\alpha)$ on $\mathcal{O}_{X}=\mathcal{O}_{X} \otimes 1$ induced by the V-filtration on the $\mathcal{D}_{X^{\prime}}$-module $B_{f}:=i_{f+} \mathcal{O}_{X}=$ $\mathcal{O}_{X} \otimes \mathbb{C}\left[\partial_{t}\right]$ along the smooth hypersurface $H: t=0$, see [7. Here $i_{f+}$ denotes the direct image as a $\mathcal{D}$-module. Therefore Theorem 1.2 is not a straightforward 
consequence of Theorem 1.1] above. We can generalize Theorem 1.2 to the case of arbitrary subvarieties (see [5.3). From Theorem 1.2 we can deduce the following application to the spectrum in the sense of J. Steenbrink 39] (see (4.4-5)):

Corollary 1.3. Under the assumption of Theorem 1.2, the spectrum $\operatorname{Sp}(f, x)$ of $f$ at $x \in T$ coincides with $\operatorname{Sp}\left(\left.f\right|_{T}, x\right)$ up to a sign. In particular, $\operatorname{Sp}\left(\left.f\right|_{T}, x\right)$ is independent of $T$ in Theorem 1.2.

It has been known that the spectrum is constant under a $\mu$-constant deformation of isolated hypersurface singularities, see [41. We have a weak generalization as follows.

Corollary 1.4. Let $S$ be a (connected) stratum of a Whitney regular stratification of $D_{\text {red. }}$. Then $\operatorname{Sp}(f, x)$ and $\operatorname{Sp}\left(\left.f\right|_{T}, x\right)$ are independent of $x \in S$, where $T$ is transversal to $S$ at $x$.

Note that the $\mu$-constantness is equivalent to the Thom $a_{f}$-condition (see [25] and 4.8 below) and the latter is weaker than the Whitney (b) condition, see 44. It is not clear whether Corollary 1.4 holds assuming only the $a_{f}$-condition without the Whitney (b) condition.

\section{BASIC FACTS ON $\mathcal{D}$-MODULES}

2.1. Non-characteristic inverse images. Let $X$ be a complex $n$-dimensional manifold and denote by $T^{*} X$ its cotangent bundle. A point $(x, \xi) \in T^{*} X$ is just a pair formed by a point $x \in X$ and a linear form $\xi: T_{x} X \rightarrow \mathbb{C}$, where $T_{x} X$ denotes the tangent space to $X$ at the point $x$. When $Z \subset X$ is a locally closed analytic subset of $X$, we denote by $T_{Z}^{*} X$ the conormal space of $Z$ in $X$. This is by definition the closure in $T^{*} X \mid Z:=\pi^{-1}(Z)$, with $\pi: T^{*} X \rightarrow X$ the canonical projection, of the set of pairs $(x, \xi) \in T^{*} X$ with $x \in Z$ a smooth point on $Z$ and $\xi \mid T_{x} Z=0$. In particular, $T_{X}^{*} X$ is just the zero section of the cotangent bundle.

Definition 2.2. Let $M$ be a coherent $\mathcal{D}_{X}$-module and let $C V(M) \subset T^{*} X$ be its characteristic variety. A submanifold $Z \subset X$ is non characteristic for $M$ if

$$
C V(M) \cap T_{Z}^{*} X \subset T_{X}^{*} X
$$

The following basic example explains the relation to the transversality discussed briefly in the Introduction.

Example 2.3. The Riemann-Hilbert correspondence, see [31, 19], says that the DR-functor establishes an equivalence of categories

$$
D R: D_{r h}^{b}\left(\mathcal{D}_{X}\right) \rightarrow D_{c}^{b}\left(\mathbb{C}_{X}\right)
$$

such that, for a regular holonomic $\mathcal{D}_{X}$-module $M$, the sheaf complex $\mathcal{F}=D R(M)$ is a perverse sheaf. Moreover, the characteristic variety $C V(M)$ coincides to the 
characteristic variety $C V(\mathcal{F})$, which is defined topologically, see for instance [21] or [10], p. 111-113. If $\mathcal{S}$ is a Whitney regular stratification of $X$ such that $\mathcal{F}$ is $\mathcal{S}$-constructible, then

$$
C V(\mathcal{F}) \subset \cup_{S \in \mathcal{S}} T_{S}^{*} X
$$

see for instance [10, p. 119. It follows that a submanifold $T$ which is transversal to $\mathcal{S}$, i.e. it is transversal to all the strata $S \in \mathcal{S}$, it is automatically non-characteristic for $M$. Note that transversality of $T$ to a single stratum $S$ at a point $x \in S$ implies, via the (a)-regularity condition, transversality to all strata in a small neighborhood of $x$ in $X$.

To be even more specific, when $M=\mathcal{O}_{X}(* D)$, then $D R\left(\mathcal{O}_{X}(* D)\right)=R j_{*} \mathbb{C}_{U}[n]$, where $U=X \backslash D$ and $j: U \rightarrow X$ is the inclusion, see [14, 33. If $\mathcal{S}$ is a Whitney regular stratification of $D$, then consider the Whitney regular stratification of $X$ given by $\mathcal{S}_{0}=\mathcal{S} \cup\{U\}$. It follows that $R j_{*} \mathbb{C}_{U}[n]$ is $\mathcal{S}_{0}$-constructible and hence, if $T$ is transversal to $\mathcal{S}$ as above, then $T$ is non-characteristic for $\mathcal{O}_{X}(* D)$.

The following result tells us that the property of being non-characteristic is preserved under small deformations.

Lemma 2.4. Let $M$ be a holonomic $\mathcal{D}_{X}$-module and $T \subset X$ a submanifold which is non-characteristic for $M$. Let $a \in T$ be any point in $T$ and $p:(X, a) \rightarrow(S, 0)$ be the germ of a submersion onto a smooth germ $(S, 0)$ such that the space germ induced by the special fiber $p^{-1}(0)$ coincides to $(T, a)$. Then there is an open neighborhood $U$ of a in $X$ on which $p$ is defined and such that all the fibers $p^{-1}(s) \cap U$ for $s \in S$ are non-characteristic for the $\mathcal{D}_{U}$-module $M \mid U$.

Proof. Since the question is local on $X$, we may suppose that $X=S \times T$ and $p$ is the first projection. On the other hand, it is known that the characteristic variety of a holonomic $\mathcal{D}_{X}$-module $M$ has the following local decomposition

$$
C V(M)=\cup_{j=1, m} T_{Z_{j}}^{*} X
$$

where $Z_{j}$ are closed irreducible analytic subsets in $X$ and the conormal spaces $T_{Z_{j}}^{*} X$ are exactly the irreducible components of the characteristic variety $C V(M)$. If a neighborhood $U$ as claimed above does not exist, then there is an index $j$ and a sequence of points $a_{n} \in X$ such that

(i) $a_{n} \rightarrow a$;

(ii) for each $n$, there is a point $\left(a_{n}, \xi_{n}\right) \in T_{Z_{j}}^{*} X$ such that $\xi_{n} \neq 0$ and $\xi_{n} \mid 0 \times T_{q\left(a_{n}\right)} T=$ 0 , where $q: S \times T \rightarrow T$ is the second projection.

Moreover, we can norm the linear form $\xi_{n}$, e.g. by dividing by their norm and passing to a convergent subsequence, such that we may assume that

(iii) $\xi_{n} \rightarrow \xi \neq 0$. 
Since $T_{Z_{j}}^{*} X$ is a closed subset, it follows that $(a, \xi)=\lim \left(a_{n}, \xi_{n}\right) \in T_{Z_{j}}^{*} X$. From (ii) we infer that $\xi \mid 0 \times T_{q(a)} T=0$. Therefore $(a, \xi) \in T_{T}^{*} X \cap C V(M)$, in contradiction to the fact that $T$ is non-characteristic for $M$.

We recall now the following result on non-characteristic inverse images, see for instance [17] or [27, Prop. II.1.3, Thm. II.1.7.

Proposition 2.5. Let $T$ be a submanifold in $X$ given by global equations $z_{1}=\ldots=$ $z_{c}=0$, where $c=\operatorname{codim} T$ such that $T$ is non-characteristic for a coherent $\mathcal{D}_{X^{-}}$ module $M$. If $i_{T}: T \rightarrow X$ is the closed inclusion of $T$ into $X$, then the derived inverse image complex $i_{T}^{+} M=\mathbb{L} i_{T}^{*} M$ is concentrated in degree zero and coincides to the coherent $\mathcal{D}_{T}$-module

$$
i_{T}^{*} M=\frac{i_{T}^{-1} M}{z_{1} i_{T}^{-1} M+\ldots+z_{c} i_{T}^{-1} M} .
$$

Moreover, $z_{1}, \ldots, z_{c}$ is a regular sequence in $i_{T}^{-1} M$ and $i_{T}^{*} M$ is holonomic when $M$ is holonomic. More precisely, in this last situation, if $C V(M)=\cup_{j=1, m} T_{Z_{j}}^{*} X$, then

$$
C V\left(i_{T}^{*} M\right)=\cup_{j=1, m} T_{T \cap Z_{j}}^{*} T .
$$

The idea of the proof of this result is to determine the characteristic variety $C V\left(i_{T}^{*} M\right)$, see Thm. II.1.7 in [27]. In the proof of Prop. II.1.3 in [27] it is shown that $\mathbb{L}_{T}^{*} M$ is represented by the Koszul complex of the sequence $z_{1}, \ldots, z_{c}$ in $M$. Then this sequence is shown to be regular, by showing that any local section germ in $M$ is killed by some special type differential operators, similar to the ones we construct below in Lemma 3.2. See also Corollary I.3.3 in 27. It follows that the Koszul complex has non-trivial homology only in degree zero, which yields the result.

2.6. V-filtrations, b-polynomials and vanishing cycles. Let $X$ be a complex analytic manifold and $H \subset X$ a smooth hypersurface. Let $\mathcal{I}$ be the ideal sheaf defining $H$. We define the decreasing Kashiwara-Malgrange $V$-filtration of $\mathcal{D}_{X}$ along $H$ by setting, for $k \in \mathbb{Z}$,

$$
V^{k}\left(\mathcal{D}_{X}\right)=\left\{P \in \mathcal{D}_{X} \mid P\left(\mathcal{I}^{j}\right) \subset \mathcal{I}^{j+k} \text { for all } j \in \mathbb{Z}\right\}
$$

where $\mathcal{I}^{j}=\mathcal{O}_{X}$ for any $j<0$. It is easy to check that $V^{k}\left(\mathcal{D}_{X}\right) \cdot V^{\ell}\left(\mathcal{D}_{X}\right) \subset V^{k+\ell}\left(\mathcal{D}_{X}\right)$. In particular, $V^{0}\left(\mathcal{D}_{X}\right)$ is a coherent sheaf of rings, see [26], Prop. 2.1.5.

If $t=0$ is a local equation for $H$, then the differential operator $t \partial_{t}$ induces an element $E$ in $G r_{V}^{0} \mathcal{D}_{X}=V^{0}\left(\mathcal{D}_{X}\right) / V^{1}\left(\mathcal{D}_{X}\right)$. This is called the Euler operator of $H$ and it is independent of the choice of the local equation $t=0$.

A coherent $\mathcal{D}_{X}$-module $M$ is said to be specializable along the hypersurface $H$ if it satisfies the following condition: 
(*) For any point $x \in H$ and any germ $m \in M_{x}$, there is a non zero polynomial $b(s) \in \mathbb{C}[s]$ such that $b(E+1) m \in V^{1}\left(\mathcal{D}_{X}\right)_{x} m$.

For more details, see [26], Propositions II.1.9, II.2.2 and II.2.4. It is known that a holonomic $\mathcal{D}_{X}$-module is specializable along any smooth hypersurface, see 18 . The Bernstein polynomial (or the b-function) $b_{m}$ of the germ $m \in M_{x}$ is the unitary polynomial of minimal degree satisfying the condition $(\star)$. Note that $E+1=\partial_{t} \cdot t$. This shift by 1 is justified by the formula (2.3) below.

Let $<$ be a total order on $\mathbb{C}$ such that, for any $u, v \in \mathbb{C}$ we have $u<u+1$, $u<v$ if and only if $u+1<v+1$ and, finally, there is some $m \in \mathbb{N}$ such that $v<u+m$. For instance, we can take the lexicografic order on $\mathbb{C}=\mathbb{R}^{2}$. Using this order, we define the decreasing Kashiwara-Malgrange $V$-filtration along $H$ on the coherent $\mathcal{D}_{X}$-module $M$, assumed to be specializable along $H$, by

$$
V^{\alpha} M_{x}=\left\{m \in M_{x} \mid \text { all the roots of the } b \text {-function } b_{m} \text { are } \geq \alpha\right\} .
$$

See Kashiwara [18], Malgrange [29], and also 34]. The filtration $V^{\alpha} M$ is indexed by a finite union of lattices $\beta+\mathbb{Z}$ in $\mathbb{C}$, hence it is a discrete, decreasing and exhaustive filtration on $M$. In most cases coming from geometry, e.g. when $M$ is obtained by applying some natural functors to the $\mathcal{D}_{X}$-module $\mathcal{O}_{X}$, then all $\beta \in \mathbb{Q}$, and hence $V$ is actually indexed by $\mathbb{Q}$. This is the case in particular for the module $M=B_{f}$. It turns out that the $\mathrm{V}$-filtration can be defined by the following list of characteristic properties, see [34], 37] for more details. Define $V^{>\alpha} M=\cup_{\beta>\alpha} V^{\beta} M$ and $G r_{V}^{\alpha} M=$ $V^{\alpha} M / V^{>\alpha} M$.

Proposition 2.7. Let $M$ be a coherent $\mathcal{D}_{X}$-module, specializable along $H$. The Kashiwara-Malgrange $V$-filtration is the unique discrete, decreasing and exhaustive filtration on $M$ satisfying the following conditions:

(1) $V^{k}\left(\mathcal{D}_{X}\right) \cdot V^{\alpha} M \subset V^{k+\alpha} M$, for any $k \in \mathbb{Z}$ and $\alpha \in \mathbb{C}$;

(2) $V^{\alpha} M$ is a coherent $V^{0}\left(\mathcal{D}_{X}\right)$-module, for any $\alpha \in \mathbb{C}$;

(3) $t \cdot V^{\alpha} M=V^{\alpha+1} M$, for $\alpha>>0$;

(4) the action of $\partial_{t} \cdot t-\alpha$ on $G r_{V}^{\alpha} M$ is locally nilpotent.

Remark 2.8. Many authors prefer to work with the increasing V-filtration defined by $V_{\alpha} M=V^{-\alpha} M$, see for instance [26]. Our choice here is justified by the fact that, as we mentioned in the Introduction, in an important case the V-filtration is essentially the filtration induced by the family of multiplier ideals, which is by definition a decreasing filtration.

Definition 2.9. Let $M$ be a regular holonomic $\mathcal{D}_{X}$-module. Let $t=0$ be a global equation for the hypersurface $H$. Then the nearby and the vanishing cycle functors $\psi_{t}, \phi_{t}: \operatorname{Mod}_{r h}\left(\mathcal{D}_{X}\right) \rightarrow \operatorname{Mod}_{r h}\left(\mathcal{D}_{H}\right)$ are defined as follows:

$$
\psi_{t} M=\oplus_{0<\alpha \leq 1} G r_{V}^{\alpha} M
$$


and

$$
\phi_{t} M=\oplus_{0 \leq \alpha<1} G r_{V}^{\alpha} M .
$$

These functors are related to the topological perverse nearby and vanishing cycle functors ${ }^{p} \psi_{t},{ }^{p} \phi_{t}: \operatorname{Perv}(X, \mathbb{C}) \rightarrow \operatorname{Perv}(H, \mathbb{C})$, see for instance [10], p. 139, by the following relations $D R \circ \psi_{t}={ }^{p} \psi_{t} \circ D R$ and $D R \circ \phi_{t}={ }^{p} \phi_{t} \circ D R$. We recall also the exact triangle in $D^{b}\left(\mathcal{D}_{H}\right)$

$$
\psi_{t} M \rightarrow \phi_{t} M \rightarrow i_{H}^{+} M \rightarrow
$$

where $i_{H}: H \rightarrow X$ is the inclusion and the morphism can $: \psi_{t} M \rightarrow \phi_{t} M$ is induced by $\partial_{t}$.

In the sequel we will regard not only $\psi_{t} M, \phi_{t} M$ but also any $G r_{V}^{\alpha} M$ as $\mathcal{D}_{T^{-}}$ modules. Let $\Psi_{t} M=i_{H+} \psi_{t} M$ and $\Phi_{t} M=i_{H+} \phi_{t} M$ be the corresponding $\mathcal{D}_{X^{-}}$ modules supported by the hypersurface $H$.

2.10. Relatively specializable $\mathcal{D}_{X}$-modules and relative $\mathbf{V}$-filtrations. In the situation of the previous subsection, suppose that we have in addition a submersion $p: X \rightarrow S$ such that the composition $p_{H}=p \circ i_{H}$ is still a submersion. We denote by $\mathcal{D}_{X / S} \subset \mathcal{D}_{X}$ the sheaf of relative differential operators, which is by definition the sheaf of subrings in $\mathcal{D}_{X}$ spanned by $\mathcal{O}_{X}$ and by the derivations coming from vector fields on $X$, tangent to the fibers of $p$. We define the relative $\mathrm{V}$-filtration on $\mathcal{D}_{X / S}$ simply by setting $V^{k}\left(\mathcal{D}_{X / S}\right)=V^{k}\left(\mathcal{D}_{X}\right) \cap \mathcal{D}_{X / S}$.

Definition 2.11. A coherent $\mathcal{D}_{X / S}$-module $M$ is relatively specializable along $H$ if there exists a decreasing, exhaustive filtration $U^{k}(M)_{k \in \mathbb{Z}}$ of $M$ by coherent $V^{0}\left(\mathcal{D}_{X / S}\right)$ submodules such that the following conditions are satisfied:

(1) $V^{k}\left(\mathcal{D}_{X / S}\right) \cdot U^{\ell}(M) \subset U^{k+\ell}(M)$, for any $k, \ell \in \mathbb{Z}$;

(2) Locally on $X$, there is an $\ell \in \mathbb{N}$ such that, for any integer $k \in \mathbb{N}$, we have $V^{k}\left(\mathcal{D}_{X / S}\right) \cdot U^{\ell}(M)=U^{k+\ell}(M)$ and $V^{-k}\left(\mathcal{D}_{X / S}\right) \cdot U^{-\ell}(M)=U^{-k-\ell}(M)$;

(3) Locally on $X$, there exists a nonzero polynomial $b(s) \in \mathbb{C}[s]$ such that $b(E-$ $k) U^{k}(M) \subset U^{k+1}(M)$, for all $k \in \mathbb{Z}$.

For more details, see [28]. In fact, one can see that if $M$ is a coherent $\mathcal{D}_{X}$-module, specializable along $H$, and such that the $V^{0}\left(\mathcal{D}_{X}\right)$-modules $V^{\alpha}(M)$ are coherent over $V^{0}\left(\mathcal{D}_{X / S}\right)$, then $M$ is relatively specializable along $H$ as a $\mathcal{D}_{X / S^{-}}$-module. Moreover, the converse implication is easy to check. In the following, we will use this last characterization.

2.12. Characteristic varieties. Let $X$ be a complex $n$-dimensional manifold, $Z \subset$ $X$ an irreducible analytic subset and $f: X \rightarrow \mathbb{C}$ a holomorphic function such that the restriction $f \mid Z$ is not a constant function, i.e. $Z$ is not contained is a fiber of $f$. 
Definition 2.13. The relative conormal space of the restriction $f \mid Z$ is the closed analytic subset in $T^{*} X$ given by

$$
T_{f \mid Z}^{*} X=\overline{\left\{(x, \xi+\lambda d f(x)) \mid(x, \xi) \in T_{Z}^{*} X, \lambda \in \mathbb{C}\right\}}
$$

where the closure is taken in $T^{*} X$. The associated Lagrangian variety of the restriction $f \mid Z$ is the subset in $T^{*} X \mid f^{-1}(0)$ given by

$$
W_{0}(f \mid Z)=T_{f \mid Z}^{*} X \cap \pi^{-1}\left(f^{-1}(0)\right)
$$

where $\pi: T^{*} X \rightarrow X$ is the canonical projection, see [4], [13].

It follows from [16], 13], Prop. 2.14.1 that $T_{f \mid Z}^{*} X$ is an $(n+1)$-dimensional subvariety in $T^{*} X$ and $W_{0}(f \mid Z)$ is a closed, conic, Lagrangian subvariety in $T^{*} X$; in particular, $\operatorname{dim} W_{0}(f \mid Z)=n$.

Let $\mathcal{Z}=\left(Z_{a}\right)_{a \in A}$ be an analytic stratification of the analytic set $Z$, i.e. $Z=$ $\cup_{a \in A} Z_{a}$ is a partition of $Z$ into smooth semianalytic subsets $Z_{a}$ satisfying the frontier condition: if $Z_{a} \cap \bar{Z}_{b} \neq \emptyset$, then $Z_{a} \subset \bar{Z}_{b}$. Similarly, let $\mathcal{T}=\left(T_{b}\right)_{b \in B}$ be an analytic stratification of $\mathbb{C}$, such that the pair $(\mathcal{Z}, \mathcal{T})$ give a stratification for $f: Z \rightarrow \mathbb{C}$, i.e. for any $a \in A$ there is a $b \in B$ such that $f\left(Z_{a}\right) \subset T_{b}$ and the induced mapping $f: Z_{a} \rightarrow T_{b}$ is a submersion.

Definition 2.14. We say that the stratification $(\mathcal{Z}, \mathcal{T})$ of $f: Z \rightarrow \mathbb{C}$ satisfies the Thom $a_{f}$-condition if for any pair of strata $Z_{a} \subset \bar{Z}_{b}$ one has

$$
T_{f \mid \bar{Z}_{b}}^{*} X \cap \pi^{-1}\left(Z_{a}\right) \subset T_{f \mid Z_{a}}^{*} X
$$

where we set $T_{f \mid S}^{*} X=T_{S}^{*} X$ whenever $f \mid S$ is a constant function.

In particular, if $\mathcal{Z}$ is a stratification of the pair $\left(Z, Z_{0}\right)$ where $Z_{0}=f^{-1}(0) \cap Z$, it follows that the induced stratification $\mathcal{Z}_{0}$ on $Z_{0}$ satisfies the Whitney $(a)$-condition. In such a case

$$
W_{0}(f \mid Z)=\cup_{S}\left(T_{f \mid Z}^{*} X \cap \pi^{-1}(S)\right) \subset \cup_{S} T_{S}^{*} X
$$

where $S$ runs through the strata of $\mathcal{Z}_{0}$. This last union is a closed subset, since this property is equivalent to the Whitney $(a)$-condition for the induced stratification $\mathcal{Z}_{0}$. See also 15 for other results about the relative conormal space and the relation with Thom's $\left(a_{f}\right)$-condition.

We recall the geometric formulation of Ginsburg results in [13, Prop. 2.14.4 and Thm. 3.3 and 5.5 given by Briançon, Maisonobe and Merle in [4], Thm 3.4.2.

Proposition 2.15. Let $M$ be a regular holonomic $\mathcal{D}_{X}$-module, $M_{0} \subset M$ a coherent $\mathcal{O}_{X}$-submodule spanning $M$ over $\mathcal{D}_{X}$, and $f: X \rightarrow \mathbb{C}$ a holomorphic function. Assume that the characteristic variety of $M$ is given by $C V(M)=\cup_{j} T_{Z_{j}}^{*} X$ as in (2.1). Then the following equalities hold, the first two ones in a neighborhood of the zero set $f=0$. 
(1) $C V\left(\mathcal{D}_{X}[s] M_{0} f^{s}\right)=\bigcup_{f \mid Z_{j} \neq 0} T_{f \mid Z_{j}}^{*} X$;

(2) $C V(M[1 / f])=\bigcup_{f \mid Z_{j} \neq 0} T_{Z_{j}}^{*} X \cup \bigcup_{f \mid Z_{j} \neq 0} W_{0}\left(f \mid Z_{j}\right)$;

(3) If $H: f=0$ is a smooth hypersurface, then by setting $t=f$, we have $C V\left(\Psi_{t} M\right)=\bigcup_{f \mid Z_{j} \neq 0} W_{0}\left(f \mid Z_{j}\right)$ in $T^{*} X$.

Here the $\mathcal{D}_{X}$-module $\mathcal{D}_{X}[s] M_{0} f^{s}$ is defined as follows. Consider the $\mathcal{O}_{X}$-submodule $M_{0} f^{s}$ in the $\mathcal{D}_{X}$-module $M[1 / f, s] f^{s}=M \otimes_{\mathcal{O}_{X}} \mathcal{O}_{X}[1 / f, s] f^{s}$, where the action of $\mathcal{D}_{X}$ on $M[1 / f, s] f^{s}$ is the usual one, e.g.

$$
\frac{\partial}{\partial x_{k}} \cdot f^{s}=s \cdot \frac{\partial f}{\partial x_{k}} \cdot 1 / f \cdot f^{s}
$$

Then $\mathcal{D}_{X}[s] M_{0} f^{s}$ is the $\mathcal{D}_{X}$-module spanned by $M_{0} f^{s}$ in $M[1 / f, s] f^{s}$, which is known to be coherent (for the case $M_{0}=\mathcal{O}_{X} \cdot m$ see [27], Lemme III.1.2).

Concerning the last property in Prop. 2.15, note that $\bigcup_{f \mid Z_{j} \neq 0} W_{0}\left(f \mid Z_{j}\right)=\cup_{k} T_{Y_{k}}^{*} X$, where $\left(Y_{k}\right)_{k}$ is a locally finite family of irreducible subvarieties in $H$. It follows that, when we regard $\psi_{t} M$, one has the decomposition

$$
C V\left(\psi_{t} M\right)=\cup_{k} T_{Y_{k}}^{*} H .
$$

Corollary 2.16. Let $X$ be a complex manifold, $H \subset X$ a smooth hypersurface given by $t=0$ and $p: X \rightarrow S$ a submersion such that the restriction $p_{H}=p \mid H$ is still a submersion. Let $M$ be a regular holonomic $\mathcal{D}_{X}$-module such that the fibers of $p$ are non-characteristic for $M$ and $M(* H)$. Then the fibers of the restriction $p_{H}$ are non-characteristic for the nearby cycle module $\psi_{t} M$, the vanishing cycle module $\phi_{t} M$ and any $\mathcal{D}_{H}$-module $G r_{V}^{\alpha} M$, for $\alpha \in \mathbb{C}$.

Proof. Let $F_{s}=p^{-1}(s)$ be a fiber of $p$ and note that $F_{s}$ is transversal to $H$. Then, using a similar formula to (2.6), we infer that, for a $\mathcal{D}_{H}$-module $N, F_{s} \cap H$ is noncharacteristic for $N$ if and only if $F_{s}$ is non-characteristic for $i_{H+} N$. Using Prop. 2.15, we get

$$
C V\left(\Psi_{t} M\right) \subset C V(M(* H)) .
$$

This implies that $F_{s}$ is non-characteristic for $\Psi_{t} M$ and hence $F_{s} \cap H$ is non-characteristic for $\psi_{t} M$. On the other hand, the exact triangle

$$
R \Gamma_{H}(M)_{\text {alg }} \rightarrow M \rightarrow M(* H) \rightarrow
$$

implies $C V\left(R \Gamma_{H}(M)_{a l g}\right) \subset C V(M) \cup C V(M(* H))$, and hence $F_{s}$ is non-characteristic for $R \Gamma_{H}(M)_{a l g}$. Since $H$ is smooth, it follows that

$$
R \Gamma_{H}(M)_{a l g}=i_{H !} i_{H}^{!}(M)
$$

see [1], 1.5.4-1.5.5. Using now the fact that a regular holonomic $\mathcal{D}_{X^{-}}$-module $M$ and its dual $D M$ have the same characteristic variety and that $D\left(i_{H !} i_{H}^{!}(M)\right)=i_{H+} i_{H}^{+} M$, it follows that $F_{s}$ is non-characteristic for $i_{H+} i_{H}^{+} M$. Using now the exact triangle (2.4), it follows that $F_{s} \cap H$ is non-characteristic for $\phi_{t} M$. 
Since $C V\left(M_{1} \oplus M_{2}\right) \supset C V\left(M_{1}\right)$, it follows from the above that $F_{s} \cap H$ is noncharacteristic for $G r_{V}^{\alpha} M$, for $\alpha \in \mathbb{C}, 0 \leq \alpha \leq 1$. The proof is completed using the fact that

$$
t: G r_{V}^{\alpha} M \rightarrow G r_{V}^{\alpha+1} M
$$

is an isomorphism of $\mathcal{D}_{H}$-modules, for any $\alpha \neq 0$.

Remark 2.17. The proof above shows that, with the above notation, for a submanifold $T$ in $X$, the condition

(C1) $T$ is non-characteristic for $M$ and $M(* H)$

is equivalent to the condition

(C2) $T$ is non-characteristic for $M$ and $i_{H+} i_{H}^{+} M$.

Lemma 2.4 and Corollary 2.16 yield the following

Corollary 2.18. Let $X$ be a complex manifold, $H \subset X$ a smooth hypersurface given by $t=0$ and $T \subset X$ a submanifold which is transverse to $H$. Let $M$ be a regular holonomic $\mathcal{D}_{X}$-module such that $T$ is non-characteristic for $M$ and $M(* H)$. Then the submanifold $T \cap H$ is non-characteristic for the nearby cycle module $\psi_{t} M$, the vanishing cycle module $\phi_{t} M$ and any $\mathcal{D}_{H}$-module $G r_{V}^{\alpha} M$, for $\alpha \in \mathbb{C}$.

\section{Proof of Theorem 1.1}

By writing $T$ as the intersection of $d$ smooth hypersurfaces, each of them transversal to $D$, we may restrict our attention to the case $d=\operatorname{dim} S=\operatorname{codim} T=1$. We start with the following general result, giving a sufficient condition for a regular holonomic module to be relatively specializable. Recall the setting and the notation from section 2.10 .

Proposition 3.1. Let $M$ be a regular holonomic $\mathcal{D}_{X}$-module such that the fibers of $p: X \rightarrow S$ are non-characteristic for $M$ and for $M(* H)$. Then $M$ is relatively specializable along the smooth hypersurface $H \subset X$.

Proof. Let $a$ be a point in $H$ and choose a local system of coordinates

$$
\left(x_{1}, \ldots, x_{n-2}, y, t\right)
$$

at $a$ in $X$ such that $h\left(x_{1}, \ldots, x_{n-2}, y, t\right)=t=0$ is a local equation for $H$ and $p\left(x_{1}, \ldots, x_{n-2}, y, t\right)=y$. To proceed, we need the following.

Lemma 3.2. With the above assumptions, for any local section germ $m \in M_{a}$ or $m \in M(* H)_{a}$, there is a differential operator $\widetilde{P} \in V^{0}\left(\mathcal{D}_{X}\right)$ killing $m$ and having the following special form

$$
\widetilde{P}=\left(\frac{\partial}{\partial y}\right)^{k}+A_{1}\left(\frac{\partial}{\partial y}\right)^{k-1}+\cdots+A_{k}
$$


where $A_{i}$ are operators of degree $\leq i$, independent of the derivatives $\partial / \partial t, \partial / \partial y$.

Proof. Let $M_{0} \subset M(* H)$ be a coherent $\mathcal{O}_{X}$-module spanning $M(* H)$ over $\mathcal{D}_{X}$. It follows then from Proposition 2.15. (i) that the fibers of $p$ are non-characteristic for the coherent $\mathcal{D}_{X}$-module $\mathcal{D}_{X}[s] M_{0} h^{s}$. In particular, for any germ $m \in M(* H)$, there is a differential operator $P$ of total degree $k$, killing $m t^{s}$, and of the form

$$
P=\left(\frac{\partial}{\partial y}\right)^{k}+A_{1}\left(\frac{\partial}{\partial y}\right)^{k-1}+\cdots+A_{k}
$$

Here the differential operators $A_{i}$ are of degree $\leq i$, independent of the derivation $\partial / \partial y$ (compare to the proof of Proposition II.1.3 in [27]).

Recall also the simple fact that an equality

$$
\sum_{i=0}^{k}\left(\frac{\partial}{\partial t}\right)^{i} m_{i} t^{s}=0
$$

with $m_{0}, \ldots, m_{k} \in M(* H)_{a}$ implies $m_{0}=\ldots=m_{k}=0$, see for instance [26] Lemme 2.4.1. Therefore, there is a differential operator $\widetilde{P}$, having the same properties as the operator $P$, and being in addition independent of $\partial / \partial t$.

To end the proof of this Lemma, we have still to consider the case of a germ $m \in M$ killed by a power of $t$. In other words, we may suppose now that $M$ is supported by the hypersurface $H$. In particular, $M$ is the image under $i_{H+}$ of a $\mathcal{D}_{H}$-module $N$ such that the fibers of $p_{T}=p \circ i_{T}$ are non-characteristic for $N$. But then the same argument as above applied to $N$ shows that the germs of sections in $N$, and hence in $M$, are killed by a differential operator similar to the operator $\widetilde{P}$, but which in this case are independent of the variable $t$ and of the derivative $\partial / \partial t$.

Let us return now to the proof of Proposition 3.1. Let $m \in M_{a}$ be the germ of a local section. Using the corresponding differential operator $\widetilde{P} \in V^{0}\left(\mathcal{D}_{X}\right)$ obtained as in the above Lemma, we get

$$
V^{\ell}\left(\mathcal{D}_{X}\right)_{a} m=\sum_{\kappa<k} V^{\ell}\left(\mathcal{D}_{X \mid S}\right)_{a}(\partial / \partial y)^{\kappa} m
$$

for all $\ell \in \mathbb{Z}$. It follows that the $V^{0}\left(\mathcal{D}_{X}\right)$-modules $V^{\alpha}(M)$ are $V^{0}\left(\mathcal{D}_{X / S}\right)$-coherents; in particular, the specializable module $M$ is relatively specializable along $H$.

Finally we can prove Theorem 1.1. Using Lemma 2.4 we can place ourselves in the relative case of a fibration $p: X \rightarrow S$ such that $T=p^{-1}(0)$ is the special fiber of $p$ and $D=H$ is the smooth hypersurface. If we regard $V^{\alpha} M$ as a $V^{0} \mathcal{D}_{X^{-}}$-module, then one has

$$
\mathbb{L} i_{T}^{*}\left(V^{\alpha} M\right)=i_{T}^{*}\left(V^{\alpha} M\right)
$$


This follows from a $V^{0} \mathcal{D}_{X^{-}}$-version of Proposition 2.5. using the differential operator constructed in Lemma 3.2 to show that the action of $y$ is injective on $M$. Note that there is a natural morphism

$$
\nu: i_{T}^{*}\left(V^{\alpha} M\right) \rightarrow i_{T}^{*}(M)
$$

induced by the inclusion $V^{\alpha} M \rightarrow M$. This morphism $\nu$ is actually injective. To see this, apply the functor $\mathbb{L} i_{T}^{*}$ to the exact sequence of $V^{0} \mathcal{D}_{X}$-modules

$$
0 \rightarrow V^{\alpha} M \rightarrow M \rightarrow \frac{M}{V^{\alpha} M} \rightarrow 0
$$

and note that the action of $y$ on the last term is injective by the same argument as above; in particular, we have

$$
\mathbb{L} i_{T}^{*} \frac{M}{V^{\alpha} M}=i_{T}^{*} \frac{M}{V^{\alpha} M}
$$

To prove that the image of the morphism $\nu$ is exactly $V^{\alpha} i_{T}^{*} M$, we proceed as follows. First, we get as above

$$
\mathbb{L} i_{T}^{*} V^{0} \mathcal{D}_{X \mid S}=i_{T}^{*} V^{0} \mathcal{D}_{X \mid S}=V^{0}\left(\mathcal{D}_{T}\right)
$$

Then, applying the functor $i_{T}^{*}=\otimes_{V^{0} \mathcal{D}_{X \mid S}} V^{0}\left(\mathcal{D}_{T}\right)$ to a presentation of $V^{\alpha} M$ as a coherent $V^{0} \mathcal{D}_{X \mid S^{-}}$module, which exists via Proposition 3.1. we get that $\nu\left(i_{T}^{*}\left(V^{\alpha} M\right)\right)$ is a coherent $V^{0}\left(\mathcal{D}_{T}\right)$-module. Since the filtration on $i_{T}^{*} M$ given by $\left(\nu\left(i_{T}^{*}\left(V^{\alpha} M\right)\right)\right)_{\alpha}$ clearly satisfies all the other conditions in Proposition [2.7] it follows that

$$
\nu\left(i_{T}^{*}\left(V^{\alpha} M\right)\right)=V^{\alpha} i_{T}^{*} M
$$

which ends the proof of the first claim in Theorem 1.1

The second claim in Theorem 1.1 follows by applying the functor $\mathbb{L} i_{T}^{*}=i_{T}^{*}$ to the exact sequence of $V^{0}\left(\mathcal{D}_{X}\right)$-modules

$$
0 \rightarrow V^{>\alpha} M \rightarrow V^{\alpha} M \rightarrow G r_{V}^{\alpha} M \rightarrow 0 .
$$

\section{Proof of Theorem 1.2}

It is clearly enough to treat the case when $T$ is a hypersurface. Since the question is local, we will assume that $X=\mathbb{C}^{n}, x_{0}=0$ is the origin. Let us choose a system of coordinates $(y, z)$ at the origin, with $y=\left(y_{1}, \ldots, y_{n-1}\right)$ such that $T: z=0$. Then the induced effective divisor $T \cap D$ is given by an equation $f_{T}(y)=f(y, 0)=0$. Consider the following diagram of closed embeddings, where $T^{\prime}=T \times \mathbb{C}$.

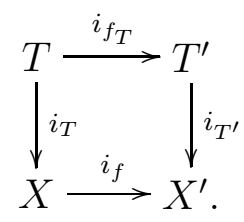


Let $t$ be a coordinate on $\mathbb{C}$ and set as in the Introduction $B_{f}=\left(i_{f}\right)_{+} \mathcal{O}_{X}=\mathcal{O}_{X} \otimes$ $\mathbb{C}\left[\partial_{t}\right]$, where $\partial_{t}=\partial / \partial t$ and $\mathcal{J}(\alpha)=V^{\alpha} B_{f} \cap\left(\mathcal{O}_{X} \otimes 1\right)$. Similarly, we set $B_{f_{T}}=$ $\left(i_{f_{T}}\right)_{+} \mathcal{O}_{T}$ and $\mathcal{J}_{T}(\alpha)=V^{\alpha} B_{f_{T}} \cap\left(\mathcal{O}_{T} \otimes 1\right)$. Using Theorem 0.1 in [7], in order to prove Theorem 1.2, it is enough to show that

$$
i_{T}^{*} \mathcal{J}(\alpha)=\mathcal{J}_{T}(\alpha)
$$

for all $\alpha \in \mathbb{Q}$.

By hypothesis $T^{\prime}$ is non-characteristic for the $\mathcal{D}_{X^{\prime}}$-modules $B_{f}$ and $B_{f}\left[\frac{1}{t}\right]$ (using the coordinate $\left.t^{\prime}:=t-f\right)$. Proposition 2.5 then yields

$$
\mathbb{L} i_{T^{\prime}}^{*} B_{f}=i_{T^{\prime}}^{*} B_{f}=B_{f_{T}}
$$

and we infer from Theorem 1.1 that

$$
i_{T^{\prime}}^{*} V^{\alpha} B_{f}=V^{\alpha} B_{f_{T}}
$$

for all $\alpha \in \mathbb{Q}$. The module $B_{f}$ is endowed with a natural increasing Hodge filtration, given up-to a shift by

$$
F_{p} B_{f}=\oplus_{0 \leq j \leq p} \mathcal{O}_{X} \otimes \partial_{t}^{j}
$$

for all $p \in \mathbb{Z}$. In particular $\mathcal{O}_{X} \otimes 1=F_{0} B_{f}$.

Using the Hodge filtration $F$, we may write

$$
\mathcal{J}(\alpha)=V^{\alpha} B_{f} \cap F_{0} B_{f}
$$

and similarly

$$
\mathcal{J}_{T}(\alpha)=V^{\alpha} B_{f_{T}} \cap F_{0} B_{f_{T}}
$$

Since obviously $i_{T^{\prime}}^{*}\left(\mathcal{O}_{X} \otimes \partial_{t}^{j}\right)=\mathcal{O}_{T} \otimes \partial_{t}^{j}$, it follows that

$$
i_{T^{\prime}}^{*}\left(F_{p} B_{f}\right) \simeq F_{p} B_{f_{T}}
$$

for all $p \in \mathbb{N}$. Hence the relation (4.1) above is equivalent to

$$
i_{T^{\prime}}^{*}\left(V^{\alpha} B_{f} \cap F_{0} B_{f}\right) \simeq i_{T^{\prime}}^{*}\left(V^{\alpha} B_{f}\right) \cap i_{T^{\prime}}^{*}\left(F_{0} B_{f}\right) .
$$

Therefore, we complete the proof of Theorem 1.2 if we prove the following

Lemma 4.1. The isomorphism

$$
i_{T^{\prime}}^{*}\left(V^{\alpha} B_{f} \cap F_{p} B_{f}\right) \simeq i_{T^{\prime}}^{*}\left(V^{\alpha} B_{f}\right) \cap i_{T^{\prime}}^{*}\left(F_{p} B_{f}\right)
$$

holds for all $p \in \mathbb{Z}$ and all $\alpha \in \mathbb{Q}$.

Set $M=B_{f}, F_{p} V^{\alpha} M=F_{p} M \cap V^{\alpha} M, V^{\alpha}\left(M / F_{p} M\right)=V^{\alpha} M / F_{p} V^{\alpha} M$ and $F_{p}\left(M / V^{\alpha} M\right)=F_{p} M / F_{p} V^{\alpha} M$. Then the following diagram, where all the morphisms are the canonical monomorphisms or canonical epimorphisms, has obviously 
exact rows and exact columns.

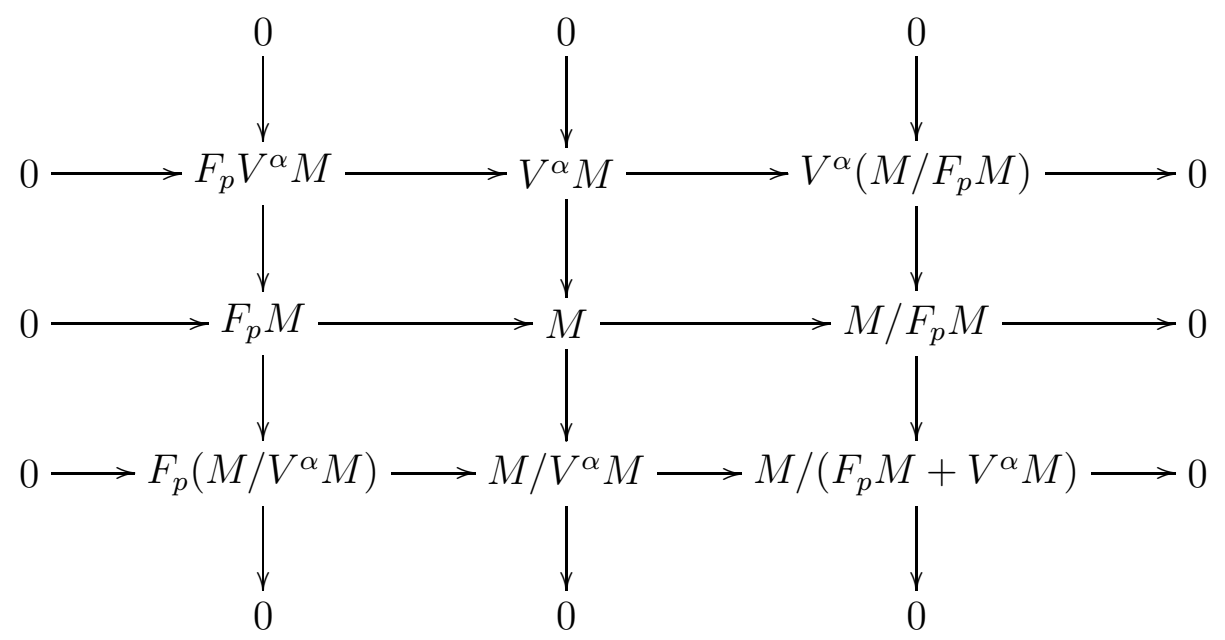

Lemma 4.1 is clearly equivalent to the following

Lemma 4.2. The diagram

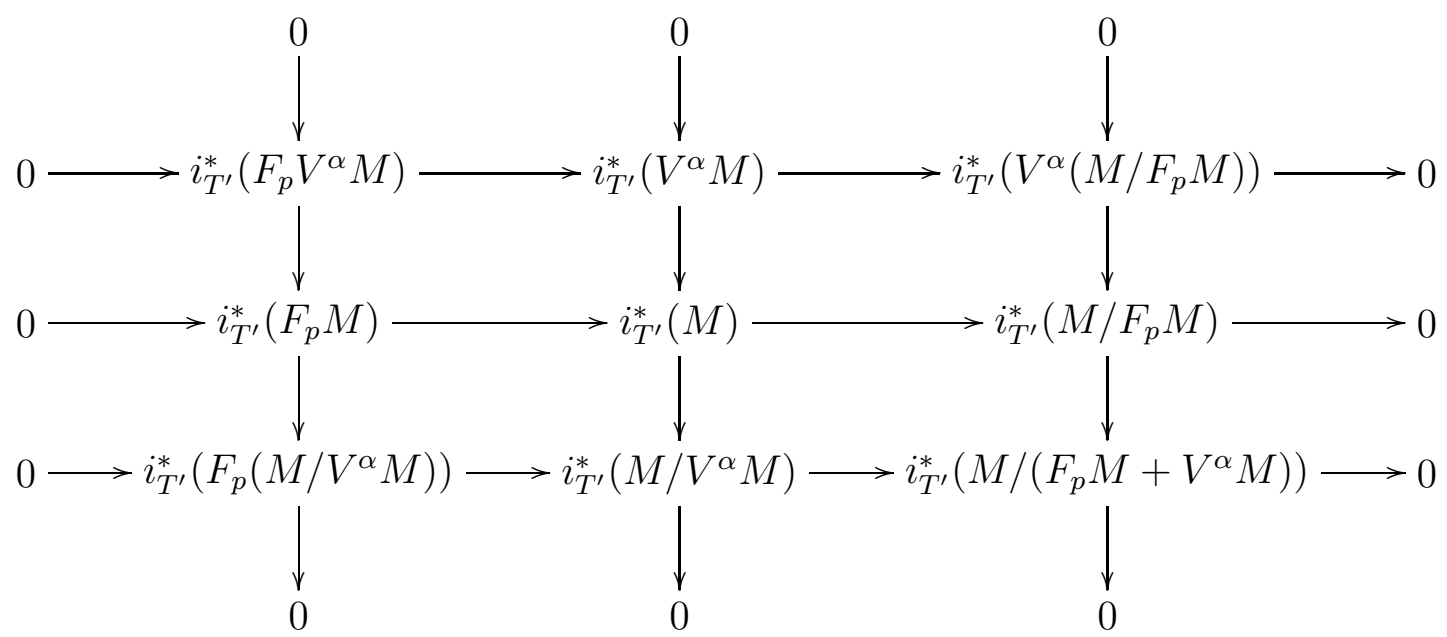

obtained from the above diagram by applying the functor $i_{T^{\prime}}^{*}$ has still exact rows and exact columns.

Now, if we have an exact sequence of $R$-modules

$$
0 \rightarrow N^{\prime} \rightarrow N \rightarrow N^{\prime \prime} \rightarrow 0
$$

and if the action of $z \in R$ is injective on $N^{\prime \prime}$, then it follows, e.g. by the use of Tor exact sequence, that the induced sequence

$$
0 \rightarrow N^{\prime} / z N^{\prime} \rightarrow N / z N \rightarrow N^{\prime \prime} / z N^{\prime \prime} \rightarrow 0
$$

is still exact. It follows that all we have to prove is in fact the following. 
Lemma 4.3. The action of $z \in \mathcal{O}_{X^{\prime}}$ is injective on any of the five $\mathcal{O}_{X^{\prime}}$-modules occurring in the third column and the third row of the diagram (4.7).

Proof. We have already proved that the action of $z$ is injective on $M / V^{\alpha} M$ (see the proof of Theorem 1.1). On the other hand, the assumption for $M / F_{p} M$ is clear since $M / F_{p} M$ is $\mathcal{O}_{X^{\prime}}$ free. Thus we just have to check the assertion for $M /\left(F_{p} M+V^{\alpha} M\right)$. In fact we prove a slightly stronger claim, i.e. we show that the action of $z$ is injective on any of the quotients

$$
F_{p} / F_{q}\left(V^{\alpha} / V^{\beta}\right) M=\frac{F_{p} V^{\alpha} M}{F_{p} V^{\beta} M+F_{q} V^{\alpha} M}
$$

for any $p>q$ in $\mathbb{Z}$ and any $\alpha<\beta$ in $\mathbb{Q}$.

Here we can use the following well-known fact:

Let $N_{1} \subsetneq N_{2} \subsetneq \cdots \subsetneq N_{p}$ be an increasing sequence of $R$-modules. Assume that the action of $z \in R$ is injective on $N_{i} / N_{i-1}$ for $2 \leq i \leq p$. Then the action of $z$ is injective on $N_{i} / N_{j}$ for $1 \leq j<i \leq p$.

Therefore it is enough to show that the action of $z$ is injective on $M_{p}^{\alpha}=G r_{p}^{F} G r_{V}^{\alpha} M$. Consider the graded $G r^{F} \mathcal{D}_{X}$-module

$$
M^{\alpha}=\oplus_{p} M_{p}^{\alpha}
$$

and note that

$$
\operatorname{supp} M^{\alpha}=C V\left(G r_{V}^{\alpha} M\right) .
$$

Suppose the action of $z$ on $M^{\alpha}$ is not injective. Then some irreducible component of supp $M^{\alpha}$ is contained in $\{z=0\}$ since $M^{\alpha}$ is a Cohen-Macaulay $G r^{F} \mathcal{D}_{X^{-}}$module (see [35, Lemme 5.1.13). Indeed, if $z m=0$ for some nonzero $m \in M^{\alpha}$, then the support of the submodule generated by $m$ is contained in $\{z=0\}$ and its dimension coincides with that of $M^{\alpha}$ by the Cohen-Macaulay property. On the other hand, $T$ is non-characteristic for $G r_{V}^{\alpha} M$ by Prop. 2.15 because $T^{\prime}$ is non-characteristic for $\mathcal{B}_{f}\left[\frac{1}{t}\right]$. This is clearly a contradiction. Thus we get the injectivity of the action of $z$. This completes the proof of Theorem 1.2

4.4. Spectrum. For a holomorphic function $f$ on a complex manifold $X$ and $x \in$ $f^{-1}(0)$, the spectrum $\operatorname{Sp}(f, x)=\sum_{\alpha \in \mathbb{Q}} m_{\alpha} t^{\alpha}$ is defined by

$$
\begin{array}{r}
m_{\alpha}=\sum_{j}(-1)^{j-n+1} \operatorname{dim} G r_{F}^{p} \widetilde{H}^{j}\left(F_{x}, \mathbb{C}\right)_{\lambda} \\
\text { with } p=[n-\alpha], \quad \lambda=\exp (-2 \pi i \alpha),
\end{array}
$$

where $n=\operatorname{dim} X, F_{x}$ denotes the Milnor fiber of $f$ around $\left.x, \widetilde{H}^{j}\left(F_{x}\right), \mathbb{C}\right)_{\lambda}$ is the $\lambda$-eigenspace of the reduced cohomology for the semi-simple part of the Milnor monodromy, and $F^{p}$ denotes the Hodge filtration, see [39] (and also [37]). 
4.5. Proof of Corollary 1.3. By the arguments in the proof of Theorem 1.2, the three filtrations $F, V_{t}, V_{z}$ on $\mathcal{B}_{f}$ are compatible in the sense of [35], 1.1.13. (Indeed, $V_{z}$ is the $z$-adic filtration in this case and applying the multiplication by $z^{j}$ to the diagram (4.7) in the proof of Lemma 4.1 we get a cubic diagram of short exact sequences which is equivalent to the compatibility of the three filtrations. Here $V_{t}$ denotes the $V$-filtration along $t=0$ and similarly for $V_{z}$.) This compatibility implies $i_{T}^{*}\left(\varphi_{f}\left(\mathcal{O}_{X}, F\right)\right)=\varphi_{f \mid T}\left(i_{T}^{*}\left(\mathcal{O}_{X}, F\right)\right)$, because $i_{T}^{*}=\psi_{z}$ and $\varphi_{z}=0$ on $\left(\varphi_{f}\left(\mathcal{O}_{X}, F\right)\right.$ in this case.

Let $i_{x}:\{x\} \rightarrow X$ denote the inclusion morphism and $i_{x}^{*}$ be the pull-back in the category of filtered $\mathcal{D}$-modules underlying mixed Hodge modules. This is defined by iterating the mapping cone of $\psi_{x_{i}, 1} \rightarrow \varphi_{x_{i}, 1}$ where the $x_{i}$ are local coordinates. Then the Hodge filtration on the Milnor cohomology is given by $H^{j} i_{x}^{*}\left(\varphi_{f}\left(\mathcal{O}_{X}, F\right)\right)$, see also [3]. So the assertion follows.

4.6. Proof of Corollary 1.4, Let $i_{S}: S \rightarrow X$ denotes the inclusion. By Proposition 2.15 the characteristic variety of the nearby cycle sheaf is contained in the union of the conormal bundles of the strata of the stratification satisfying the Thom $a_{f}$-condition (see 2.14). Therefore the $\mathcal{H}^{j} i_{S}^{*} \psi_{f} \mathbb{C}_{X}$ for $j \in \mathbb{Z}$ are local systems by 21, Proposition 8.4.1 (note that a $\mu$-stratification in loc. cit. is Whitney regular, see Trotman [40]). Since the pull-back by $i_{S}$ is compatible with the pull-back in the derived category of mixed Hodge modules, these local systems naturally underly variations of mixed Hodge structures. (Note that a mixed Hodge module is a variation of mixed Hodge structure if its underlying perverse sheaf is a local system up to a shift of complex). So the Hodge numbers are constant, and we get the assertion for $\operatorname{Sp}(f, x)$. Then the assertion for $\operatorname{Sp}\left(\left.f\right|_{T}, x\right)$ follows from Corollary 1.3.

Remark 4.7. It is not clear whether Corollary 1.4 holds assuming only the $a_{f^{-}}$ condition. Note that for $K \in D_{c}^{b}\left(\mathbb{C}_{X}\right)$ and a stratification satisfying only the Whitney $(a)$ condition and such that the characteristic variety $C V(K)$ of $K$ is contained in the union of the conormal bundles of the strata of the stratification, the restriction of $H^{j} K$ to each stratum is not locally constant in general. For example, let $D=\left\{f:=x^{3}+x^{2} z^{2}-y^{2}=0\right\} \subset \mathbb{C}^{3}$, and consider the stratification of $D$ defined by $S=\{x=y=0\}, S^{\prime}=D \backslash S$. An easy direct verification shows that this stratification is (a)-regular. The singularities of $D$ are resolved by the blow-up $\rho: D^{\prime} \rightarrow D$ along $S$ and there are coordinates $u, v$ of $D^{\prime}$ such that $\rho^{*} x=u^{2}-v^{2}, \rho^{*} y=u^{3}-u v^{2}, \rho^{*} z=v$ with $u=\rho^{*}(y / x)$. Note that $\rho$ is the normalization and $\rho^{-1}(0)$ consists of a point $0^{\prime}$. Let $K=\rho_{*} \mathbb{C}_{D^{\prime}}$. Then $\operatorname{rank} K_{x}=2$ if and only if $x \in S \backslash\{0\}$. In particular, $\left.K\right|_{S}$ is not a local system. On the other hand, the dimension of $C V(K) \cap T_{0}^{*} X$ is at most 2 by the estimation of the characteristic variety of the direct image (see [21] or [10], 4.3.3) because the rank of $d \rho$ at $0^{\prime}$ is 1 . For $x \in S \backslash\{0\}, C V(K) \cap T_{x}^{*} X$ consists of two lines spanned respectively by $d g_{1}$ 
and $d g_{2}$ where $g_{1}, g_{2}$ are functions defining the local irreducible components of $D$ at $x$. Therefore $C V(K)$ is the closure of the conormal bundle of $S^{\prime}$ by the involutivity of characteristic varieties (see e.g. 21]).

Remark 4.8. The above example does not give a $\mu$-constant deformation when $z$ is viewed as a parameter, and the $a_{f}$-condition is not satisfied for $f$. In general, it is known that the converse of a result of 25 holds, i.e. for a holomorphic function $f$ defined on a neighborhood $X$ of the origin of $\mathbb{C}^{n} \times \mathbb{C}^{r}$, the restriction $f_{t}$ of $f$ to $X \cap \mathbb{C}^{n} \times\{t\}$ is $\mu$-constant if the stratification of $D=\{f=0\} \subset X$ defined by $S=X \cap\{0\} \times \mathbb{C}^{r}$ and $S^{\prime}=D \backslash S$ satisfies the $a_{f}$-condition (assuming $S^{\prime}$ smooth). This easily follows from the fact that $f_{t}$ has a critical point at $x \neq 0$ if and only if the tangent space of $\{f=a\}$ at $(x, t)$ contains $\mathbb{C}^{n} \times\{0\}$ where $a=f_{t}(x)$ and $(x, t) \in X$ is sufficiently near 0 .

\section{Generalization of Theorem 1.2}

5.1. Deformation to the normal cone. Let $X$ be a complex manifold, and $Z$ be a closed submanifold. Let

$$
\mathcal{X}=\operatorname{Specan}_{X}\left(\bigoplus_{i \in \mathbb{Z}} I_{Z}^{-i} \otimes t^{i}\right)
$$

where $I_{Z}$ is the ideal sheaf of $Z$ in $X$ and $I_{Z}^{-i}=\mathcal{O}_{\mathcal{X}}$ for $i \geq 0$. Note that $\mathcal{X}$ is naturally identified with an open subset of the blow-up of $X \times \mathbb{C}$ along $Z \times\{0\}$. We have the projection $p: \mathcal{X} \rightarrow \mathbb{C}$ defined by $t$, and $p^{-1}\left(\mathbb{C}^{*}\right)=X \times \mathbb{C}^{*}$. Therefore $\mathcal{X}$ gives a deformation of $X$ to the normal cone $N_{Z / X}$ of $Z$ in $X$ (see [42]) because $N_{Z / X}$ is isomorphic to

$$
p^{-1}(0)=\operatorname{Specan}_{Z}\left(\bigoplus_{i \leq 0}\left(I_{Z}^{-i} / I_{Z}^{-i+1}\right) \otimes t^{i}\right) .
$$

Let

$$
\mathcal{A}=\left.\bigoplus_{i \in \mathbb{Z}} I_{Z}^{-i}\right|_{Z} \otimes t^{i}
$$

This is identified with a subsheaf of $\left.\mathcal{O}_{\mathcal{X}}\right|_{Z}$ where $Z$ is identified with the zero section of $N_{Z / X}=p^{-1}(0) \subset \mathcal{X}$. Note that the stalk $\mathcal{A}_{x}$ at each $x \in Z$ is noetherian because there is a surjective ring morphism $\mathcal{O}_{X, x}\left[t_{0}, \ldots, t_{r}\right] \rightarrow \mathcal{A}_{x}$ sending $t_{0}$ to $1 \otimes t$ and $t_{i}$ to $x_{i} \otimes t^{-1}$ if we take local coordinates $x_{i}$ of $X$ such that $x_{i}$ for $1 \leq i \leq r$ generate the ideal of $Z$. Moreover, $\mathcal{A}_{x}$ has a regular sequence consisting of $1 \otimes t, x_{i} \otimes t^{-1}$ for $1 \leq i \leq r$, and $x_{i} \otimes 1$ for $i>r$. Therefore its completion by the maximal ideal generated by these elements is isomorphic to the ring of formal power series over $\mathbb{C}$. This implies that $\mathcal{O}_{\mathcal{X}, x}$ is flat over $\mathcal{A}_{x}$ because the completion is faithfully flat over $\mathcal{O}_{\mathcal{X}, x}$, and is flat over $\mathcal{A}_{x}$. 
5.2. Specialization. Let $j: X \times \mathbb{C}^{*} \rightarrow \mathcal{X}$ denote the natural inclusion, and $p r$ : $X \times \mathbb{C}^{*} \rightarrow X$ the natural projection. For a regular holonomic $\mathcal{D}_{X}$-module $M$, consider the open direct image $j_{+} p r^{*} M$ of the smooth pull-back $p r^{*} M$ in the category of regular holonomic $\mathcal{D}$-modules. Note that $j_{+} p r^{*} M$ is the localization by $t$ of the pull-back $\rho^{*} M$ of $M$ by the natural morphism $\rho: \mathcal{X} \rightarrow X$, and

$$
\left.\rho^{*} M\right|_{Z}=\left.\left(\left.\bigoplus_{i \in \mathbb{Z}}\left(M \otimes_{\mathcal{O}_{X}} I_{Z}^{-i}\right)\right|_{Z} \otimes t^{i}\right) \otimes_{\mathcal{A}} \mathcal{O}_{\mathcal{X}}\right|_{Z}
$$

Therefore $\left.\left(j_{+} p r^{*} M\right)\right|_{Z}$ is naturally isomorphic to

$$
\left(\left.\bigoplus_{i \in \mathbb{Z}} M\right|_{Z} \otimes t^{i}\right)^{\sim}:=\left.\left(\left.\bigoplus_{i \in \mathbb{Z}} M\right|_{Z} \otimes t^{i}\right) \otimes_{\mathcal{A}} \mathcal{O}_{\mathcal{X}}\right|_{Z}
$$

Note that $\partial_{t}$ acts on $\left.\left(j_{+} p r^{*} M\right)\right|_{Z}$, and the kernel of $t \partial_{t}-i$ is identified with $\left.M\right|_{Z} \otimes t^{i}$, where $\partial_{t}$ is the vector field on $X \times \mathbb{C}^{*}$ associated with the coordinate $t$ of $\mathbb{C}^{*}$ together with the product structure of $X \times \mathbb{C}^{*}$.

Let $V$ be the filtration of Kashiwara and Malgrange on $M$ along $Z$, and similarly for $j_{+} p r^{*} M$ along $p^{-1}(0)$. The specialization of $M$ along $Z$ is defined by

$$
\psi_{t} j_{+} p r^{*} M
$$

Let $r=\operatorname{codim}_{X} Z$. It is known (see [6] for the algebraic case) that

$$
\left.V^{\alpha-r+1}\left(j_{+} p r^{*} M\right)\right|_{Z}=\left(\left.\bigoplus_{i \in \mathbb{Z}} V^{\alpha-i} M\right|_{Z} \otimes t^{i}\right)^{\sim}
$$

where $\mathcal{M}^{\sim}$ for an $\mathcal{A}$-module $\mathcal{M}$ in general is defined by $\left.\mathcal{M} \otimes_{\mathcal{A}} \mathcal{O}_{\mathcal{X}}\right|_{Z}$. This isomorphism follows by showing that the filtration defined by the right-hand side satisfies the conditions of the $V$-filtration of Kashiwara and Malgrange. For the proof of this, we can omit the tensor with $\left.\mathcal{O}_{\mathcal{X}}\right|_{Z}$ over $\mathcal{A}$ because this is an exact functor.

Assume that $M$ underlies a mixed Hodge module on $X$. In particular, $M$ has the Hodge filtration $F$. The Hodge filtration on the smooth pull-back $p r^{*} M$ is given by $p r^{*} F$, see [36]. Let $p_{0}=\min \left\{p \mid F_{p} M \neq 0\right\}$. Then

$$
\left.F_{p_{0}}\left(j_{+} p r^{*} M\right)\right|_{Z}=\left(\left.\bigoplus_{i \in \mathbb{Z}} F_{p_{0}} V^{r-1-i} M\right|_{Z} \otimes t^{i}\right)^{\sim},
$$

because $F_{p_{0}}\left(j_{+} p r^{*} M\right)=V^{0}\left(j_{+} p r^{*} M\right) \cap j_{*} F_{p_{0}}\left(p r^{*} M\right)$, see 35 . Note that $F_{p_{0}} V^{-i} M=$ $F_{p_{0}} M$ for $i \gg 0$. Since the tensor with $\left.\mathcal{O}_{\mathcal{X}}\right|_{Z}$ over $\mathcal{A}$ is an exact functor and commutes with intersections of submodules, we get for $\alpha \geq r-1$

$$
\left.F_{p_{0}} V^{\alpha-r+1}\left(j_{+} p r^{*} M\right)\right|_{Z}=\left(\left.\bigoplus_{i \in \mathbb{Z}} F_{p_{0}} V^{\alpha-i} M\right|_{Z} \otimes t^{i}\right)^{\sim} .
$$

Therefore $\left.F_{p_{0}} V^{\alpha-i} M\right|_{Z}$ is obtained from $\left.F_{p_{0}} V^{\alpha-r+1}\left(j_{+} p r^{*} M\right)\right|_{Z}$ by restricting to the kernel of $t \partial_{t}-i$.

Theorem 5.3. Let $D$ be a subvariety of $X$ which is not necessarily reduced nor irreducible. Let $f=\left(f_{1}, \ldots, f_{r}\right)$ be a system of generators of the ideal of $D$, and $i_{f}: X \rightarrow X \times \mathbb{C}^{r}$ be the graph embedding by $f$. Assume $T \times \mathbb{C}^{r}$ is non-characteristic for the specialization of the $\mathcal{D}$-module $i_{f+} \mathcal{O}_{X}$ along $X \times\{0\}$, where the normal bundle of $X \times\{0\}$ in $X \times \mathbb{C}^{r}$ is identified with $X \times \mathbb{C}^{r}$. Then the assertion of Theorem 1.2 holds for $D$. 
Proof. We reduce the assertion to the divisor case applying the above argument to $M=i_{f+} \mathcal{O}_{X}$ and $Z=X \times\{0\} \subset X \times \mathbb{C}^{r}$. Note that the arguments in Section 4 apply to the case of any mixed Hodge modules including the situation in this section. Note that the vector field $\partial_{t}$ is defined by using the product structure of $X \times \mathbb{C}$, and induces a vector field on $T \times \mathbb{C}$. Here we may assume that $T$ is defined by a coordinate $z$ of $X$. Then, in order to reduce the assertion to the divisor case as above, it is sufficient to show that the kernel of $t \partial_{t}-i_{0}$ and cokernel of the action of $z$ commute on

$$
\mathcal{M}^{\sim}:=\left(\left.\bigoplus_{i \in \mathbb{Z}} F_{p_{0}} V^{\alpha-i} M\right|_{Z} \otimes t^{i}\right)^{\sim} .
$$

Indeed, if we take first the cokernel of the action of $z$ we get the intersection of $F$ and $V$ for modules over $T$ by the proof of Theorem 1.2 ,

Let $x \in T \times\{0\} \subset \mathcal{X}$ where $\mathcal{X}$ is an open subvariety of the blow-up of $X \times \mathbb{C}^{r} \times \mathbb{C}$ along $X \times\{0\}$ and is identified with $X \times \mathbb{C}^{r} \times \mathbb{C}$. Using the snake lemma applied to

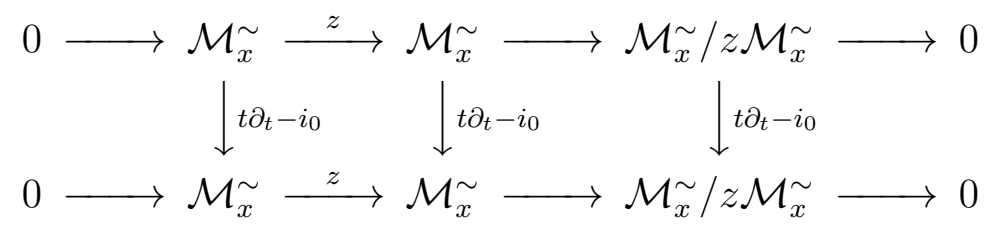

the above commutativity is reduced to the injectivity of the action of $z$ on the cokernel of $t \partial_{t}-i_{0}$ on $\mathcal{M}_{x}^{\sim}$, and to the canonical isomorphism

$$
\operatorname{Coker}\left(t \partial_{t}-i_{0}: \mathcal{M}_{x}^{\sim} \rightarrow \mathcal{M}_{x}^{\sim}\right)=\mathcal{M}_{x}^{i_{0}}
$$

where $\mathcal{M}^{i}=\left.F_{p_{0}} V^{\alpha-i} M\right|_{Z}$. This is further reduced to the surjectivity of the action of $t \partial_{t}-i_{0}$ on

$$
\prod_{i \neq i_{0}}\left(\mathcal{M}_{x}^{i} \otimes t^{i}\right) \bigcap \mathcal{M}_{x}^{\sim}
$$

Here $\mathcal{M}_{x}^{\sim}$ is viewed as a completion of $\mathcal{M}_{x}$ in some topology, and is identified with a vector subspace of $\prod_{i}\left(\mathcal{M}_{x}^{i} \otimes t^{i}\right)$. This surjectivity is reduced to the case $\mathcal{M}=\mathcal{A}$ and $\mathcal{M}^{\sim}=\left.\mathcal{O}_{\mathcal{X}}\right|_{Z}$ taking homogeneous generators $v_{j}$ of $\mathcal{M}_{x}$ (i.e. $v_{j} \in \mathcal{M}_{x}^{i_{j}} \otimes t^{i_{j}}$ ). For $\mathcal{M}=\mathcal{A}$ we take local coordinate system $\left(x_{1}^{\prime}, \ldots, x_{n}^{\prime} ; y_{1}^{\prime}, \ldots, y_{r}^{\prime} ; t^{\prime}\right)$ of $\mathcal{X}$ which is related to a local coordinate system $\left(x_{1}, \ldots, x_{n} ; y_{1}, \ldots, y_{r} ; t\right)$ of $X \times \mathbb{C}^{r} \times \mathbb{C}$ by $x_{i}=x_{i}^{\prime}, y_{j}=y_{j}^{\prime} t^{\prime}, t=t^{\prime}$. Then the vector field $t \partial_{t}$ on $\mathcal{X}$ is expressed by $t^{\prime} \partial_{t^{\prime}}-$ $\sum_{j} y_{j}^{\prime} \partial_{y_{j}^{\prime}}$, and we can prove the surjectivity using the grading of $\mathbb{C}\left\{x^{\prime}, y^{\prime}, t^{\prime}\right\}$ such that $\operatorname{deg} x_{i}^{\prime}=0, \operatorname{deg} y_{j}^{\prime}=-1, \operatorname{deg} t^{\prime}=1$. Therefore the assertion is reduced to the divisor case for arbitrary mixed Hodge modules as above, and follows from the proof of Theorem 1.2. This completes the proof of Theorem 5.3.

For the moment, it is not clear whether the assertion of Theorem 5.3 holds under the assumption that $T$ is transversal to any stratum of a Whitney regular stratification of $D$ in the case $D$ is reduced. 
A. DIMCA, PH. MAISONOBE, M. SAITO, AND T. TORRELLI

\section{REFERENCES}

[1] Briançon J., Granger M., Maisonobe Ph., Sur les systèmes différentiels relativement spécialisables et l'existence d'équations fonctionnelles relatives, Bull. Soc. Math. France 124 (1996), 217-242.

[2] Briançon J., Laurent Y., Maisonobe Ph., Sur les modules différentiels holonomes réguliers, cohérents relativement à une projection, C.R. Acad. Sci. Paris 313 (1991) 285-288.

[3] Briançon J., Maisonobe Ph., Caractérisation géométrique de l'existence du polynôme de Bernstein relatif, Progr. Math. 134, Birkhäuser, Basel (1996) 215-236.

[4] Briançon J., Maisonobe Ph, Merle M., Localisation de systèmes différentiels, stratifications de Whitney et condition de Thom, Invent. Math. 117 (1994) 531-550.

[5] Briançon J., Maisonobe Ph, Merle M., Constructibilité de l'idéal de Bernstein, Adv. Stud. Pure Math., 29, (1998) 79-95.

[6] Budur, N., MustaţǍ, M. And Saito, M.,Bernstein-Sato polynomials of arbitrary varieties(math.AG/0408408).

[7] Budur N. AND SAIto M., Multiplier ideals, $V$-filtration, and spectrum, J. Algebraic Geom. 14 (2005), 269-282.

[8] Demailly J.-P., Multiplier ideal sheaves and analytic methods in algebraic geometry, ICTP Lecture Notes vol.6, Trieste, 2001.

[9] Dimca A., Singularities and Topology of Hypersurfaces. Universitext, Springer, Berlin Heidelberg New York (1992).

[10] Dimca A., Sheaves in Topology, Universitext, Springer, Berlin 2004.

[11] Dimca A., MaAref F., Sabbah C. and Saito M., Dwork cohomology and algebraic $\mathcal{D}$-modules, Math. Ann. 318 (2000), 107-125.

[12] Gibson, C.G., Wirthmüller, K., Du Plessis, A.A., Looijenga, E.J.N., Topological Stability of Smooth Mappings. Lecture Notes in Math., 552, Springer, Berlin Heidelberg New York (1976)

[13] Ginsburg, V., Characteristic varieties and vanishing cycles, Invent. Math., 84, 327-402 (1986).

[14] Grothendieck A., On the de Rham cohomology of algebraic varieties, Pub. Math. I.H.E.S. 29 (1966) 95-105.

[15] Henry, J. P.; Merle, M.; Sabbah, C., Sur la condition de Thom stricte pour un morphisme analytique complexe, Ann. Sci. cole Norm. Sup. 17 (1984), 227-268.

[16] Kashiwara M., B-functions and holonomic systems, Invent. math. 38 (1976) 33-53.

[17] Kashiwara M., Systems of microdifferential equations, Birkhäuser, Boston, 1983.

[18] Kashiwara M., Vanishing cycle sheaves and holonomic systems of differential equations, Lecture Notes in Math. 1016 (1983) 134-142.

[19] Kashiwara M., The Riemann-Hilbert problem for holonomic systems, Publ. RIMS, Kyoto Univ. 20 (1984) 319-365.

[20] Kashiwara M., Kawai T., Second Microlocalisation and Asymptotics Expensions, Lect. Notes in Physics 126 (1980) 21-76.

[21] Kashiwara M., Schapira P., Sheaves on manifolds, Springer-Verlag, Berlin, 1990.

[22] Kollár J., Singularities of pairs. Algebraic geometry-Santa Cruz 1995, 221-287, Proc. Sympos. Pure Math., 62, Part 1, Amer. Math. Soc., Providence, RI, 1997.

[23] Lazarsfeld R., Positivity in Algebraic Geometry, vol. 2, Springer 2004.

[24] Laurent Y., Malgrange B., Cycles proches, spécialisation et D-modules, Ann. Inst. Fourier 45 (1995) 1353-1405. 
[25] LÊ D.T., Saito, K., La constance du nombre de Milnor donne des bonnes stratifications, C. R. Acad. Sci. Paris Sér. A-B 277 (1973), A793-A795.

[26] Maisonobe Ph., Mebkhout Z., Le théorème de comparaison pour les cycles évanescents, Séminaires et congrès 8, Soc. Math. France, Paris (2004) 311-389.

[27] Maisonobe Ph., Torrelli T., Image inverse en théorie des $\mathcal{D}$-Modules, Séminaires et congrès 8 , Soc. Math. France, Paris (2004) 1-57.

[28] Maisonobe Ph., Torrelli T., D-Modules relatifs et cycles évanescents, in 'Singularités', Institut Élie Cartan, Nancy (2005).

[29] Malgrange B., Polynôme de Bernstein-Sato et cohomologie évanescente, Astérisque 101-102 (1983) 233-267.

[30] Mebkhout Z., Une équivalence de catégories. Une autre équivalence de catégories, Compositio Math. 51 (1984) 51-88.

[31] Mebkhout Z., Le formalisme des six opérations de Grothendieck pour les $\mathcal{D}_{X}$-modules cohérents, Travaux en Cours 35, Hermann, Paris, (1989).

[32] Mebkhout Z., Sabbah C. D-modules et cycles évanescents in Le formalisme des six opérations de Grothendieck pour les $\mathcal{D}_{X}$-modules cohérents, Travaux en Cours 35 , Hermann, Paris, (1989) 201-238.

[33] Mebkhout Z., Le théorème de positivité, le théorème de comparaison et le théorème d'existence de Riemann, Séminaires et Congrès 8, Soc. Math. France, Paris (2004) 165-310.

[34] Sabbah C., D-modules et cycles évanescents (d'après B. Malgrange et M. Kashiwara), Géométrie algébrique et applications, III (La Rábida, 1984), Travaux en Cours 24, Hermann, Paris (1987) 53-98.

[35] Saito M., Modules de Hodge polarisables, Publ. RIMS, Kyoto Univ. 24 (1988), 849-995.

[36] Saito M., Mixed Hodge Modules, Publ. RIMS, Kyoto Univ. 26 (1990), 221-333.

[37] Saito M., On b-function, spectrum and rational singularity, Math. Ann. 295 (1993), 51-74.

[38] Schürmann, J., Topology of Singular Spaces and Constructible Sheaves. Monografie Matematyczene, New Series, Polish Academy, Birkhäuser, Basel (2003).

[39] Steenbrink, J.H.M., The spectrum of hypersurface singularity,Astérisque 179-180 (1989), $163-184$.

[40] Trotman, D., Une version microlocalede la condition (w) de Verdier, Ann. Inst. Fourier 39 (1989) 825-829.

[41] Varchenko, A. N., The complex singularity index does not change along the stratum = const, Funktsional. Anal. i Prilozhen. 16 (1982), 1-12.

[42] Verdier, J.-L., Spécialisation de faisceaux et monodromiemodérée, Analysis and topology on singular spaces, II,III (Luminy, 1981), Astérisque 101-102 (1983), 332-364. 
Laboratoire J.A. Dieudonné, UMR du CNRS 6621, Université de Nice-Sophia Antipolis, Parc Valrose, 06108 Nice Cedex 02, FRANCE.

E-mail address: dimca@math.unice.fr

Laboratoire J.A. Dieudonné, UMR du CNRS 6621, Université de Nice-Sophia ANtipolis, Parc Valrose, 06108 Nice Cedex 02, FRANCE.

E-mail address: phm@math.unice.fr

RIMS KYоTо UNIVERSITY, КYоTо 606-8502 JAPAN

E-mail address: msaito@kurims.kyoto-u.ac.jp

Laboratoire J.A. Dieudonné, UMR du CNRS 6621, Université de Nice-Sophia Antipolis, Parc Valrose, 06108 Nice Cedex 02, FRANCE.

E-mail address: torrelli@math.unice.fr 